\title{
Previous Stenting Doesn't Affect Outcome of Extensive Endarterectomy for Diffuse Coronary Artery Total Occlusions: A Propensity Score Matching Analysis
}

\author{
Hamdy Singab, $\mathbf{P h D},{ }^{1}$ Gamal Sami, $\mathrm{PhD}^{1,2}$ \\ ${ }^{1}$ Department of Cardiovascular and Thoracic Surgery, Faculty of Medicine, Ain Shams University, Cairo; \\ ${ }^{2}$ Cardiothoracic Surgery, Nasser Institute for Research and Treatment, Cairo, Egypt
}

\section{ABSTRACT}

Background and aim: Extensive diffuse coronary artery total occlusions (CTOs) constitute a challenging surgical problem. Extensive coronary endarterectomy (CE) combined with $C A B G$ was suggested as a revascularization technique. It was claimed that previous stenting may affect outcome. The present study aimed to report the outcome of LAD endarterectomy for CTO and to determine the effect of previous stenting on this outcome.

Patients and methods: The present retrospective study was conducted on 194 patients with CTO indicated for left anterior descending artery (LAD) endarterectomy. To reduce the bias related to unbalanced patients selection, propensity score matching analysis was used. According to the propensity score, 194 patients were included in the analysis. They comprised 66 patients with previous stent and 128 patients without previous stent. Patients were followed for a median (range) of 74.0 (6.0-149.0) months. The primary study outcome was LAD graft patency. Other outcome parameters included postoperative complications, hospital and ICU stay, and mortality.

Results: Comparison between the studied groups regarding outcome parameters revealed no significant differences regarding graft patency $(93.9 \%$ versus $89.1 \% ; P=.27)$, graft survival (median [95\% CI]: 134.3 months [127.0-141.5] versus 135.2 months [128.4-142.0]; $P=.35$ ), patients' survival (93.9\% versus $91.4 \%$ ) and patients' survival time (median [95\% CI]: 132.3 months [125.0-139.5] versus 138.0 months [132.0-144.1]; $P=.75]$.

Conclusion: The present study supports using CE as an adjuvant technique with CABG in patients with TCOs.

\section{INTRODUCTION}

Coronary artery total occlusions (CTOs) constitute a fairly common and clinically risky condition that represents

Received November 17, 2020; accepted Fanuary 15, 2021.

Correspondence: Hamdy Singab, PhD, Department of Cardiac Surgery, Faculty of Medicine, Ain Shams University, Abbasia Square, P.O. Box 11517, Cairo, Egypt; +201001008859; fax: +2022604465 (e-mail: hamdi_singab@hotmail. com; drhamdy-ahmed@med.asu.edu.eg). an advanced stage of stable coronary artery disease (CAD). Extensive diffuse CTOs are defined as $100 \%$ coronary occlusions persisting more than 3 months [Allahwala 2019; Koelbl 2018]. In spite of the significant technological advances and the cumulative expertise of surgical teams, management of extensive diffuse CTOs remains one of the most challenging issues in revascularization surgery, leaving a high proportion of patients untreated [Anantha-Narayanan 2019; Dash 2016].

Conventionally, the condition is treated with coronary artery bypass grafting (CABG) or medical therapy. However, evidence supporting use of CABG in management of extensive diffuse CTOs is controversial, with some authors limiting its value to cases with multivessel disease as an element of complete revascularization strategy [Weintraub 2016; Zakkar 2016].

Alternatively, percutaneous coronary intervention (PCI) has been recommended by many observational studies as an effective and relatively safe option that can be used alone or in conjunction with medical therapy [Khatri 2017; Sathananthan 2017]. Unfortunately, the success rates of PCI in management of extensive diffuse CTO remain low. In addition, the procedure is technically demanding and requires high surgical expertise even with CTO crossing algorithms [Brilakis 2016]. Moreover, there is a subset of extensive diffuse CTO cases where PCI attempts usually fail due to failure to cross the lesion [Dash 2016].

Trying to reduce the risks and improve survival of patients with extensive diffuse CTO, other surgical approaches combining two surgical techniques were advocated. Among these approaches, performing extensive coronary endarterectomy (CE) with reconstruction of the left anterior descending (LAD) artery was recognized as a useful adjunctive technique to CABG for revascularization of CTO [Katselis 2017; Myers 2012; Nardi 2018]. However, the effect of the procedure on the long-term outcomes and survival are controversial [Wang 2019]. Moreover, nothing is known about the influence of previous stenting on the operative outcome of endarterectomy for extensive diffuse CTO. It's claimed that stenting is related to pathological and structural changes that may not only affect the stented area but can also extend to the distal coronary segments [Massoudy 2009].

The present study aimed to report the outcome of LAD endarterectomy for CTO and to determine the effect of previous stenting on this outcome. 


\section{PATIENTS AND METHODS}

\section{Study Design and Participants}

The present retrospective study was conducted at the Department of Cardiothoracic Surgery, Ain Shams University, Cairo, Egypt, in the period from January 2008 through May 2018. The study protocol was approved by the local institutional ethics committee and was conducted in accordance with the Helsinki Declaration, and all patients gave informed consent before the surgical procedures. All patients' baseline preoperative data, intraoperative data, postoperative data, and follow-up data were collected from the medical records and Ain Shams University hospitals database according to the guidelines of the Society of Thoracic Surgeons Adult Cardiac database. Follow-up data were collected at our center's outpatient cardiac surgery and cardiology clinics.

\section{Selection Criteria}

According to the propensity matching score, 194 patients were included in our study analysis out of 315 patients undergoing CABG with extensive diffuse LAD CTO indicated for left anterior descending artery (LAD) endarterectomy at our center from January 2008 through May 2018. To reduce the bias related to unbalanced patients selection, propensity score matching analysis was used. The propensity matching score was calculated using logistic regression analysis. The variables included in the regression model were age, sex, preoperative NYHA classification, site of LAD patch, and type of endarterectomy. According to the propensity score, 194 patients were included in the analysis. They comprised 66 patients with previous stent and 128 patients without previous stent.

The study included 194 patients with CTO indicated for left anterior descending artery (LAD) endarterectomy. This is clinically judged by failure to pass $1-\mathrm{mm}$ probe through the affected artery intraoperatively and preoperatively with angiographic finding of extensive diffuse LAD CTO. We did not perform LAD endarterectomy for nonviable myocardium.

\section{Data Collection}

For our patients, we collected baseline preoperative clinical data: age, sex, body mass index, cardiovascular risk factors, comorbidities (hypertension, diabetes mellitus, smoking, dyslipidemia, renal failure, history of peripheral arterial disease [PAD], history of cerebrovascular disease [CVD], chronic obstructive pulmonary disease [COPD]), New York Heart Association (NYHA) classification, congestive heart failure, type of angina, angina Canadian Cardiovascular Society (CSS) grading, echocardiography, laboratory data and coronary angiography data. We recorded the patients' preoperative number of diseased vessels and previous percutaneous intervention (PTCA); and operative variables (emergency procedure, site of LAD patch, length of LAD patch, cross clamp time, total cardiopulmonary [CPB] bypass time, type of coronary endarterectomy [classical or island]).

We also recorded the patients' postoperative multislice computed tomography (MSCT) coronary angiography at 6 months, 24 months, and 48 months postoperatively for LAD graft patency as well as the postoperative echocardiography data including ejection fraction. For all patients, we collected postoperative variables (intensive care unit [ICU] stay, hospital stay, deep strenal wound infection, mechanical ventilation time, atrial fibrillation $[\mathrm{AF}]$, transient heart block, supraventricular tachycardia [SVT] and ventricular fibrillation [VF] cardioversion, hemodialysis and reoperation for postoperative bleeding).

\section{Surgical Procedure}

All patients had median sternotomy and under cardiopulmonary bypass (CPB), all patients had coronary artery bypass grafting, and coronary endarterectomy was done in case of extensive diffuse CTO with antegrade intermittent blood cardioplegia. Most coronary endarterectomy was planned preoperatively but the final decision was taken intraoperative when we failed to pass $1-\mathrm{mm}$ probe through the affected artery. Liberal coronary arteriotomy was done initially; then the occluding plaque was identified and mobilized leaving the coronary adventia in its place. In case we found an area of healthy intima in between the atherosclerotic plaque, we left this island of healthy intima (island endarterectomy) in place to promote the regeneration process and angogensis. Coronary endarterectomy was extended to allow extraction of the atherosclotics plaque core. After extraction, remaining tissue debris was washed away by injection of retrograde cardioplegic solution. Visualization of the solution through the diagonal and septal branches was a sign of successful procedure. The coronary artery bed was washed with heparinized saline to remove any debris. Onlay patch anastomosis using arterial (left internal mammary artery [LIMA] radial artery) or saphenous vein patch was performed over the extended coronary arteriotomy with $6 / 0$ or $7 / 0$ polypropylene running sutures. CABG was performed using appropriate venous or arterial grafts applied to the endarterectomized LAD as appropriate. LIMA was the most desired conduit for the endarterectomized LAD in all our cases.

\section{Antithrombotic Regimen}

All patients received dual antiplatelets (aspirin and clopidogrel). Aspirin (Bayer Bitterfeld, Germany) 100 mg/day was started 6 hours after surgery then clopidogrel (Plavix, Sanofi Pharma Bristol-Myers Squibb SNC, France) $75 \mathrm{mg} /$ day on the next day. The clopidogrel was continued for 4-6 months, and aspirin for life.

\section{Study Outcome}

Patients were followed for a median (range) of 74.0 (6.0149.0) months. The primary study outcome was LAD graft patency. Other outcome parameters included postoperative complications, hospital and ICU stay, and mortality.

\section{Statistical Analysis}

Data obtained from the present study were presented as mean \pm standard deviation (SD), median and range or number and percent. Numerical data were compared using t test or Mann-Whitney $U$ test while categorical data were compared using Pearson chi-square test or Fisher exact test. KaplanMeier survival analysis was used to represent graft patency and patients' mortality. Long-rank test was used to compare survival functions. All statistical operations were processed using 
SPSS 26, and a $P$ value of less than .05 was considered statistically significant. The propensity matching score was calculated using logistic regression analysis. The variables included in the regression model were age, sex, preoperative NYHA classification, site of LAD patch, and type of endarterectomy.

\section{RESULTS}

The present study included 194 patients subjected to LAD endarterectomy. They comprised 66 patients $(34.0 \%)$ with previous stenting and 128 patients without stenting (66.0\%). Comparison between patients with previous stenting and patients without regarding the preoperative data revealed no statistically significant differences (Table 1). Also, comparison between the studied groups regarding the operative characteristics is shown in Table 2.

Postoperatively, no significant differences were detected between the studied groups regarding postoperative NYHA classification, ejection fraction, postoperative complications, mechanical ventilation duration, and ICU stay duration.
However, it was found that patients with a previous stent had a significantly shorter hospital stay when compared to patients without $(9.0 \pm 2.3$ versus $10.2 \pm 3.6$ days; $P=.005)$ (Table 3$)$.

Comparison between the studied groups regarding outcome parameters revealed no significant differences regarding graft patency $(93.9 \%$ versus $89.1 ; P=.27)$, graft patency survival with freedom of surgical or cardiological intervention (median [95\% CI]: 134.3 months [127.0-141.5] versus 135.2 months [128.4-142.0]; $P=.35$ ), patients' survival $(93.9 \%$ versus $91.4 \%$ ) and patients' survival time (median [95\% CI]: 132.3 months [125.0-139.5] versus 138.0 months [132.0144.1]; $P=.75$ ) (Table 4; Figures 1 and 2).

\section{DISCUSSION}

In patients with TCOs, coronary endarterectomy as an adjuvant technique to CABG has the advantages of securing complete myocardial revascularization and prevention of residual ischemia. It's not surprising that the procedure is increasingly recognized as an effective and safe procedure in

Table 1. Preoperative Characteristics in Patient Groups

\begin{tabular}{|c|c|c|c|c|}
\hline & $\begin{array}{c}\text { All patients } \\
n=194\end{array}$ & $\begin{array}{c}\text { With stent } \\
n=66\end{array}$ & $\begin{array}{l}\text { Without stent } \\
\quad n=128\end{array}$ & $P$ \\
\hline Age $(y)$, mean $\pm S D$ & $53.9 \pm 6.1$ & $52.9 \pm 8.5$ & $54.4 \pm 4.4$ & .20 \\
\hline Male/female, $\mathrm{n}$ & $176 / 18$ & $58 / 8$ & $118 / 10$ & .33 \\
\hline $\mathrm{BMI}(\mathrm{kg} / \mathrm{m} 2)$, mean $\pm \mathrm{SD}$ & $32.4 \pm 9.4$ & $31.1 \pm 11.7$ & $33.0 \pm 7.9$ & .24 \\
\hline \multicolumn{5}{|l|}{ Comorbidities/risk factors, n (\%) } \\
\hline Hypertension & $114(58.8)$ & $45(68.2)$ & $69(53.9)$ & .056 \\
\hline Diabetes mellitus & $113(58.2)$ & $43(65.2)$ & $70(54.7)$ & .16 \\
\hline Smoking & $70(36.1)$ & $18(27.3)$ & $52(40.6)$ & .067 \\
\hline Dyslipidemia & $112(58.2)$ & $36(54.5)$ & $76(59.4)$ & .52 \\
\hline Renal failure & $8(4.1)$ & $3(4.5)$ & $5(3.9)$ & .83 \\
\hline History of PAD & $8(4.1)$ & $2(3.0)$ & $6(4.7)$ & .58 \\
\hline History of CVD & $2(1.0)$ & - & $2(1.6)$ & .31 \\
\hline COPD & $8(4.1)$ & $6(9.1)$ & $2(1.6)$ & .012 \\
\hline \multicolumn{5}{|l|}{ Cardiac characteristics } \\
\hline Type of angina (stable/unstable), $n$ & $154 / 40$ & $53 / 13$ & $101 / 27$ & .82 \\
\hline Ejection fraction (\%), mean \pm SD & $57.2 \pm 10.5$ & $58.2 \pm 8.5$ & $55.5 \pm 13.5$ & .15 \\
\hline NYHA $(2 / 3 / 4), n$ & $19 / 90 / 85$ & $4 / 31 / 31$ & $15 / 59 / 54$ & .44 \\
\hline Angina CSS grading $(1 / 2 / 3 / 4), n$ & $12 / 81 / 90 / 11$ & $2 / 29 / 31 / 4$ & $10 / 52 / 59 / 7$ & .62 \\
\hline Number of diseased vessels, mean \pm SD & $2.9 \pm 0.7$ & $3.0 \pm 0.7$ & $2.9 \pm 0.7$ & .63 \\
\hline Critical proximal RCA, n (\%) & $13(6.7)$ & $3(4.5)$ & $10(7.8)$ & .39 \\
\hline Congestive heart failure, $\mathrm{n}(\%)$ & $9(4.6)$ & $4(6.1)$ & $5(3.9)$ & .5 \\
\hline Previous PTCA, $\mathrm{n}(\%)$ & $2(1.0)$ & $2(3.0)$ & - & .048 \\
\hline
\end{tabular}

Values are presented as number of patients and percentage, mean and standard deviation. BMI indicates body mass index; CAD, coronary artery disease; PAD, peripheral arterial disease; CVD, cerebrovascular disease; COPD, chronic obstructive pulmonary disease; CSS, Canadian Cardiovascular Society. 
Table 2. Operative Characteristics in Patient Groups

\begin{tabular}{lccc}
\hline & $\begin{array}{c}\text { All patients } \\
\mathrm{n}=194\end{array}$ & $\begin{array}{c}\text { With stent } \\
\mathrm{n}=66\end{array}$ & $\begin{array}{c}\text { Without stent } \\
\mathrm{n}=128\end{array}$ \\
\hline $\begin{array}{l}\text { Emergency procedure, } \mathrm{n}(\%) \\
\text { Site of LAD patch, } \mathrm{n}(\%)\end{array}$ & $9(4.9)$ & $3(4.5)$ & $6(4.7)$ \\
$\quad$ Mid and distal LAD & & & \\
$\quad$ Distal LAD & $165(85.1)$ & $58(87.9)$ & $107(83.6)$ \\
Mid LAD & $27(13.9)$ & $8(12.1)$ & $2(14.8)$ \\
Length of LAD patch (cm), mean \pm SD & $2(1.0)$ & $-96)$ & $7.3 \pm 2.9$ \\
Type of endarterectomy, $\mathrm{n}(\%)$ & $7.4 \pm 2.8$ & $7.7 \pm 2.7$ & $95(74.2)$ \\
$\quad$ Classical & $138(71.1)$ & $43(65.2)$ & $33(25.8)$ \\
Island & $56(28.9)$ & $23(34.8)$ & $66.2 \pm 9.1$ \\
Cross clamp time (min), mean \pm SD & $66.5 \pm 8.7$ & $67.0 \pm 7.8$ & .32 \\
Total CPB time (min), mean \pm SD & $89.7 \pm 11.8$ & $88.7 \pm 11.5$ & $90.2 \pm 12.1$ \\
\hline
\end{tabular}

Values are presented as number of patients and percentage, mean and standard deviation.

Table 3. Postoperative Data in Patient Groups

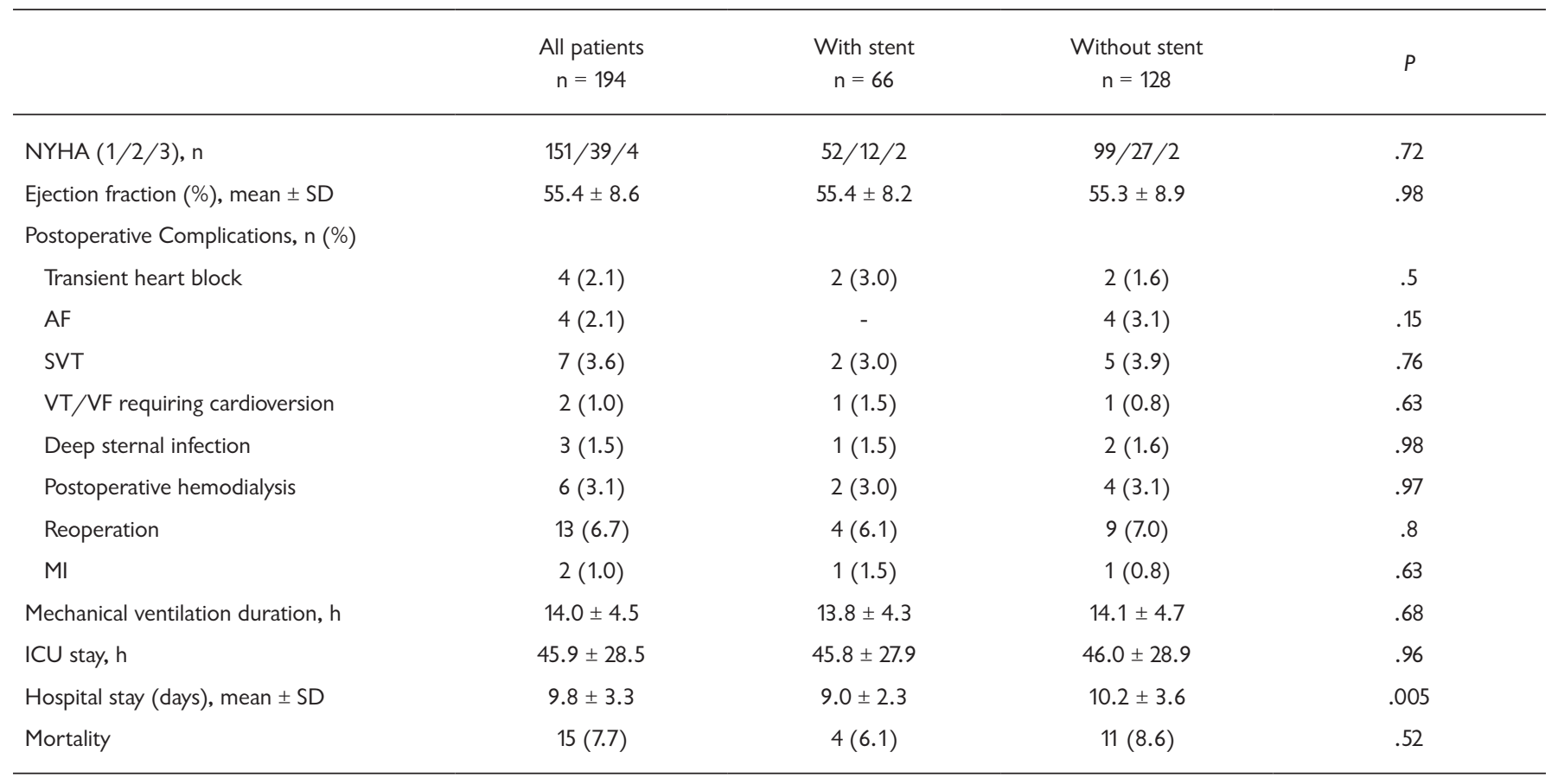

Values are presented as number of patients and percentage, mean and standard deviation. NYHA indicates New York Heart Association class; AF, atrial fibrillation; SVT, supraventricular tachycardia; VT, ventricular tachycardia; VF, ventricular fibrillation; MI, myocardial infarction.

this population [Stavrou 2016]. However, the outcome of this intervention may be affected by many clinical and technical factors [Song 2017].

As previously discussed, previous stenting may pose a challenging risk for patients with TCOs indicated for CABG plus LAD-CE due to the probable underlying inflammatory and degenerative changes. So the present study aimed to evaluate the effect of stenting on outcome of these patients. In general, the total outcome of the studied cohort was fairly good after a median follow-up of 74 months (range: 6.0-149.0 months). The overall patency rate was $90.7 \%$ while the mortality rate was $92.3 \%$. 
Table 4. Treatment Outcome in Patient Groups

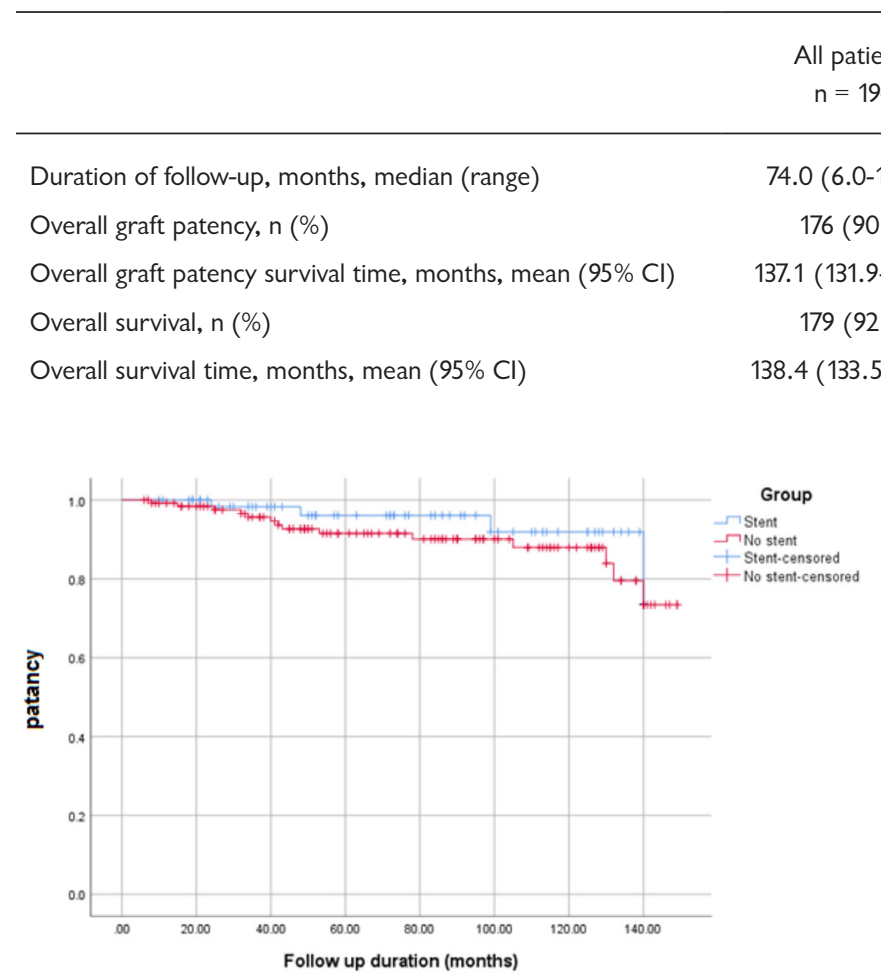

Figure 1. Kaplan-Meier curve for the long-term LAD graft patency after extensive endarterectomy for coronary artery total occlusions with non previous stenting versus coronary artery total occlusions with previous stenting.

In comparison, the 5 -year survival rate was $74.0 \%$ in the study of Byrne et al [Byrne 2004] on LAD while the study of Nurozler et al [Nurozler 2006] on LAD, right coronary artery, or other vessels reported a survival rate of $93.0 \%$ over a follow-up of $14 \pm 3.3$ months. In the study by Vohra et al [Vohra 2006], involving right coronary artery, LAD, or both, $90 \%$ of patients were angina-free with an actuarial 10-year survival rate of $78.04 \pm 7.6 \%$ after a median follow-up of 4.91 years.

The reported actuarial patency rate in the study by Sachweh et al [Sachweh 2007] was $100 \%, 96 \%$, and $56 \%$ after 2, 5, and 10 years. Favorable outcomes were reported by other studies [Katselis 2017; Binsalamah 2014; Nemati 2015; Nishigawa 2017; Takahashi 2013] regardless of the type of graft [Myers 2012], use of pump [Qiu 2014], or site of CE [Zhu 2019]. Conclusions of these studies and others were summed up in one meta-analysis that recognized a comparable long-term outcome between CE + CABG and isolated CABG groups [Wang 2015].

In spite of the potential risk related to previous stenting on patients subjected to $\mathrm{CABG}$ plus $\mathrm{CE}$, few reports addressed this issue. Fukui et al [Fukui 2005] reported their experience on CE of LAD in patients with in-stent restenosis (ISR) involving diffuse target vessel disease. Their 11-patient study concluded that $\mathrm{CE}$ and stent removal with on-lay left internal mammary artery patch in such patients is a safe and effective procedure. Another study confirmed the feasibility and safety

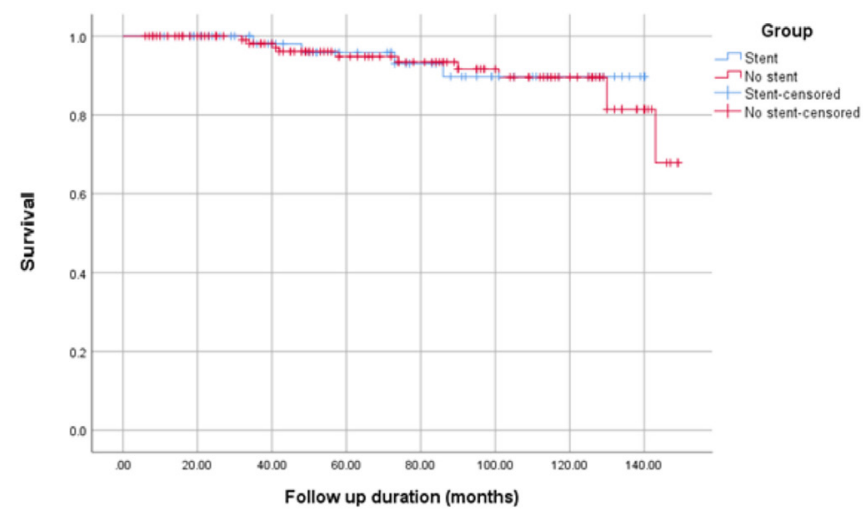

Figure 2: Kaplan-Meier curve for the long-term survival after extensive endarterectomy for coronary artery total occlusions with non previous stenting versus coronary artery total occlusions with previous stenting.

of the procedure using an off-pump [Nemati 2015]. Of note, these studies didn't compare the short and long-term operative outcome of the studied patients to others without ISR.

Interestingly, our findings revealed comparable graft patency rate, graft survival, mortality rate and patients' survival in patients with previous stenting and in patients without. These findings are supported by the recent work of ElGamel and Chan [El-Gamel 2020], which concluded that open stent CE is a reliable treatment option in patients with full metal jacket not-graftable LAD.

\section{CONCLUSION}

Our present study supports use of CE as an adjuvant technique with CABG in patients with TCOs.

\section{REFERENCES}

Allahwala UK et al. 2019. Indications for percutaneous coronary intervention (PCI) in chronic total occlusion (CTO): have we reached a DECISION or do we continue to EXPLORE after EURO-CTO? Heart, Lung and Circulation 28:1484-9.

Anantha-Narayanan M, Garcia S. 2019. Contemporary approach to 
chronic total occlusion interventions. Current Treatment Options in Cardiovascular Medicine 21:1.

Binsalamah ZM et al. 2014. Mid Term outcome and angiographic follow up of endarterectomy of the left anterior descending artery in patients undergoing coronary artery bypass surgery. J Cardiac Surg: Including Mechanical and Biological Support for the Heart and Lungs 29:1-7.

Brilakis ES et al. 2016. Update on coronary chronic total occlusion percutaneous coronary intervention. Interventional Cardiology Clinics 5:177-86.

Byrne JG et al. 2004. Left anterior descending coronary endarterectomy: early and late results in 196 consecutive patients. Ann Thoracic Surg 78:867-73.

Dash D. 2016. Complications encountered in coronary chronic total occlusion intervention: prevention and bailout. Indian heart journal 68:737-46.

El-Gamel A, Chan B. 2020. Full metal jacket endarterectomy of left anterior descending coronary artery is safe with good midterm outcomes. Heart Lung Circ S1443-9506:30439-X.

Fukui T, Takanashi S, Hosoda Y. 2005. Coronary endarterectomy and stent removal in patients with in-stent restenosis. Ann Thorac Surg 79:558-63.

Katselis C et al. 2017. Outcomes after a left anterior descending artery endarterectomy in advanced coronary artery disease. Cardiovascular Revascularization Medicine 18:332-7.

Khatri J, Abdallah M, Ellis S. 2017. Management of coronary chronic total occlusion. Cleve Clin J Med 84:27-38.

Koelbl CO, Nedeljkovic ZS, Jacobs AK. 2018. Coronary chronic total occlusion (CTO): a review. Reviews in cardiovascular medicine 19:33-9.

Massoudy P et al. 2009. Impact of prior percutaneous coronary intervention on the outcome of coronary artery bypass surgery: a multicenter analysis. J Thorac Cardiovasc Surg 137:840-5.

Myers PO et al. 2012. Extensive endarterectomy and reconstruction of the left anterior descending artery: early and late outcomes. J Thorac Cardiovasc Surg 143:1336-40.

Nardi P et al. 2018. Coronary endarterectomy: an old tool for patients currently operated on with coronary artery bypass grafting. Long-term results, risk factor analysis. Kardiochirurgia i Torakochirurgia Polska (Polish Journal of Cardiothoracic Surgery) 15:219.

Nemati MH, Astaneh B, Khosropanah S. 2015. Outcome and graft patency in coronary artery bypass grafting with coronary endarterectomy. Korean J Thorac Cardiovasc Surg 48:13.

Nishigawa K et al. 2017. Ten-year experience of coronary endarterectomy for the diffusely diseased left anterior descending artery. Ann
Thorac Surg 103:710-16.

Nurozler F et al. 2006. Off-pump coronary endarterectomy in high-risk patients. Asian Cardiovasc Thoracic Ann 14:227-30.

Qiu $Z$ et al. 2014. Comparison of off-pump and on-pump coronary endarterectomy for patients with diffusely diseased coronary arteries: early and midterm outcome. J Cardiothorac Surg 9:1-8.

Sachweh J et al. 2007. Left anterior descending coronary artery: longterm angiographic results of CABG with endarterectomy. J Cardiovasc Surg 48:633.

Sathananthan J, Džavík V. 2017. Coronary intervention for chronic total occlusion: current indications and future directions. Coronary artery disease 28:426-36.

Song Y et al. 2017. Coronary endarterectomy with coronary artery bypass graft decreases graft patency compared with isolated coronary artery bypass graft: a meta-analysis. Interactive Cardiovasc Thorac Surg 25:30-6.

Stavrou A et al. 2016. Coronary endarterectomy: the current state of knowledge. Atherosclerosis 249:88-98.

Takahashi $M$ et al. 2013. Early and mid-term results of off-pump endarterectomy of the left anterior descending artery. Interactive cardiovascular and thoracic surgery. 16:301-5.

Vohra HA et al. 2006. Early and late outcome after off-pump coronary artery bypass graft surgery with coronary endarterectomy: a single-center 10-year experience. Ann Thorac Surg 81:1691-6.

Wang J et al. 2015. Short- and long-term patient outcomes from combined coronary endarterectomy and coronary artery bypass grafting: a meta-analysis of 63,730 patients (PRISMA). Medicine (Baltimore) 94:e1781.

Wang C et al. 2019. Analysis of survival after coronary endarterectomy combined with coronary artery bypass grafting compared with isolated coronary artery bypass grafting: a meta-analysis. Interactive cardiovascular and thoracic surgery. 29:393-401.

Weintraub WS, Garratt KN. 2016. Should chronic total occlusion be treated with coronary artery bypass grafting? Chronic total occlusion should not routinely be treated with coronary artery bypass grafting. Circulation 133:1818-25.

Zakkar M, George SJ, Ascione R. 2016. Should chronic total occlusion be treated with coronary artery bypass grafting? Chronic total occlusion should be treated with coronary artery bypass grafting. Circulation 133:1807-16.

Zhu P et al. 2019. Does the site of coronary endarterectomy have an impact on the clinical outcomes and graft patency? Interactive Cardivasc Thorac Surg 29:402-8. 